\title{
Trophoblast-Targeted Nanomedicine Modulates Placental sFLT1 for Preeclampsia Treatment
}

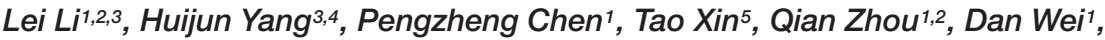 \\ Yanan Zhang ${ }^{2}$ and Shan Wang ${ }^{1,2 *}$ \\ 1 Department of Obstetrics and Gynecology, Shandong Provincial Hospital Affiliated to Shandong University, Jinan, China, \\ ${ }^{2}$ Department of Obstetrics and Gynecology, Shandong Provincial Hospital Affiliated to Shandong First Medical University, \\ Jinan, China, ${ }^{3}$ Key Laboratory of Birth Regulation and Control Technology of National Health and Family Planning \\ Commission of China, Jinan, China, ${ }^{4}$ Maternal and Child Health Care of Shandong Province, Jinan, China, ${ }^{5}$ Department \\ of Neurosurgery, The First Affiliated Hospital of Shandong First Medical University, Jinan, China
}

The overexpressed soluble fms-like tyrosine kinase 1 (sFLT-1) in placenta is considered to be a potential therapeutic target for preeclampsia (PE). How to achieve efficient intervention of sFLT1 expression in the placenta is an urgent problem to be solved. PEG-PLA nanoparticle generated by double-emulsion methods is a novel siRNA delivery system. Synthetic placental CSA binding peptide (P-CSA-BP) is effective for targeting lipid-polymer nanoparticle to the placenta. We conjugated P-CSA-BP to the surface of PEG-PLA nanoparticle to create a novel placenta specific sFLT1 siRNA delivery system for the therapy of PE. Nanoparticles were synthesized using double emulsion method and characterized by dynamic light scattering and transmission electron microscopy (TEM). RT-PCR was employed to evaluate mRNA level and protein level was analyzed by ELISA kit. The tissue distribution of nanoparticles was observed through ex vivo images. The concentrations of nanoparticles in organs were measured using high-performance liquid chromatography. T-NP sisFLT1 had higher efficiency than $\mathrm{NP}_{\text {sis } F L T 1}$ in accumulating in HTR-8/SVneo cells and significantly decreased the expression of SFLT1. Intravenously

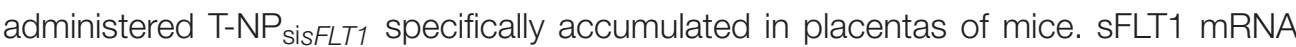
level in placenta and protein level in serum were declined by T-NP sisFLT1. T-NP $_{\text {sisFLT1 }}$ shown no obvious toxic effect on both mother and fetus. The utility of T-NPsisFLT1 nanoparticles as a sFLT1 siRNA placenta specific delivery system significantly silenced sFLT1 in mice and is safe for both mother and fetus. This nanoparticle is a novel potential therapeutic strategy for PE.

Keywords: nanoparticles, placental CSA binding peptide, sFLT1, preeclampsia, therapeutic strategy

\section{INTRODUCTION}

Preeclampsia is a special type of hypertensive disorders complicating pregnancy (HDCP) which is characterized by hypertension, proteinuria, hematological complications, and uteroplacental dysfunction (von Dadelszen and Magee, 2014). The fetal development is also significantly influenced by the pathogenesis of PE, such as amniotic fluid reduction, oxygenation defect, and

Abbreviations: sFLT-1, fms-like tyrosine kinase 1; P-CSA-BP, Synthetic placental CSA binding peptide; PE, preeclampsia. 
growth restriction (Douglas and Redman, 1994). During the pathogenesis of $\mathrm{PE}$, the dysfunction in maternal vascular system and the abnormal activation of maternal-fetal immune system are proved to be the two main factors (Laresgoiti-Servitje, 2013). The worldwide prevalence of PE in all pregnant women is from 3 to $8 \%$ and nearly $18 \%$ maternal deaths are caused by $\mathrm{PE}$ based on its high maternal morbidity and mortality (Anderson et al., 2012). The primary prevention of PE is mainly performed through the treatment of aspirin, vitamin $\mathrm{D}$, and low-dose calcium (Henderson et al., 2014). The termination of pregnancy or fetus delivery is thought to be the only definitive treatment for PE (World Health Organization [WHO], 2011). Although there has been great progress in the study of $\mathrm{PE}$, the prediction and prevention of $\mathrm{PE}$ development is still a big challenge.

$\mathrm{PE}$ is proved to be an anti-angiogenic state. Soluble fms-like tyrosine kinase 1 (sFLT-1) is a shorter form of FLT-1 which lacks the intra-cytoplasmic and transmembrane domain and thus can circulates freely in maternal circulation (Salahuddin et al., 2007). The ability of binding with vascular endothelial growth factor (VEGF) and placental growth factor (PlGF) makes sFLT1 to be the decoy receptor of these two factors in the maternal circulation (Rana et al., 2012). The sFLT1:PlGF ratio is proved to fluctuate during the normal pregnant. sFLT1 stays at a stable level till week 29-30 and then increases to a peak at week 40 (Palm et al., 2011). Meanwhile, The sFLT1:PlGF ratio decreases in week 9-12, stays at a low level from week 19-20 to 3738, and then increases in week 39-40 (Palm et al., 2011). The tightly regulated sFLT1:PlGF ratio during pregnant is crucial for both placenta and fetal development. The elevated sFLT1 level is observed in patients with PE (Levine et al., 2004). The pathogenesis of PE triggers the increasing of sFLT1 level in maternal circulation (Herraiz et al., 2018). The up-regulated sFLT-1 level in the maternal circulation decrease the levels of VEGF and PlGF and generates the endothelial dysfunction (Levine et al., 2006). It has been reported that sFLT-1 expression is up-regulated in the placenta and generated by villous and extravillous trophoblasts (Ahmad and Ahmed, 2004). Therefore, the overexpressed sFLT1 in placenta is considered to be a potential therapeutic target for PE. How to achieve efficient intervention of sFLT1 expression in the placenta is an urgent problem to be solved.

In recent, it has been proved that the systemically delivered sFLT1 siRNAs which are cholesterol conjugated and full chemical stabilized can accumulate in placentas, inhibit the expression of sFLT1, and alleviate proteinuria and hypertension (Turanov et al., 2018). Chondroitin sulfate A (CSA) is a protein which is proved to exist on the surface of placental syncytiotrophoblasts and P-CSA-BP is effective for targeting lipid-polymer nanoparticle to the placenta (Zhang et al., 2018). This research indicates that sFLT1-siRNA-based PE therapy is a hopeful therapeutic strategy. The discovery of P-CSA-BP makes it possible to develop a placenta-specific sFLT1-siRNA delivery method, which is thought to be a great progression in the improvement of sFLT1-siRNA-based PE therapeutic strategy.

In this research, we built up P-CSA-BP-conjugated nanoparticles loaded with sFLT1 siRNA to generate a new placenta-specific siRNA delivery method. We aimed to illustrate the effects of this method in placenta targeting and silencing of sFLT1 and analyze the toxic effect.

\section{MATERIALS AND METHODS}

\section{Animals}

Pregnant CD1 mice were obtained from Beijing Vital River Laboratory Animal Technology Co., Ltd. (Beijing, China). Mice were caged in pathogen-free animal room with $12 \mathrm{~h}$ light/12 h dark cycle. The gestation of mice was determined by checking vaginal plugs formation (E0.5 = vaginal plug day). At 14 and 15 days of gestation, the mice were treated by siRNA, $\mathrm{NP}_{\text {siRNA, }}$ or $\mathrm{T}-\mathrm{NP}_{\text {siRNA }}$ through intravenous injection in the tail vein at the siRNA dosage of $2 \mathrm{mg} / \mathrm{kg}$. The accumulation of Cy5-labeled sisFLT1 in mice placenta was observed through ex vivo images. Placenta tissue samples and blood samples were collected for further investigation. The procedures of animal experiments in this research were approved by the Shandong Provincial Hospital Affiliated to Shandong University.

\section{Cell Culture}

HTR-8/SVneo cell lines were obtained from the American Type Culture Collection (ATCC, VA, United States). RPMI-1640 medium (Gibco, New York, United States) with 10\% fetal bovine serum (FBS) (Gibco) and 1\% penicillin/streptomycin (Sigma, St. Louis, United States) was applied for the cell culture under the standard conditions.

\section{Preparation and Characterization of Nanoparticles}

Lipid-polymer nanoparticles loaded with sFLT1 siRNAs $\left(\mathrm{NP}_{\text {sisFLT1 }}\right)$ were synthesized from carboxyl-polyethylene glycol-poly(D,L-lactide) (COOH-PEG $\left.5 \mathrm{~K}-\mathrm{PLA}_{8 \mathrm{~K}}\right)$, cationic lipid DOTAP (N-[1-(2,3-dioleoyloxy)propyl]-N,N,N-trimeth ylammonium chloride), and sFLT1 siRNAs using double emulsion method which was previously published in other research (Xu et al., 2016). Synthetic placental CSA-binding peptides (P-CSA-BP) were conjugated to $\mathrm{NP}_{\text {sisFLT1 }}$ to generate the trophoblast-targeted nanoparticles $\left(\mathrm{T}-\mathrm{NP}_{\text {sisFLT1 }}\right)$ based on the method from other study (Zhang et al., 2018).

Size distribution and zeta potential of nanoparticles were measured through dynamic light scattering (DLS) in triplicate with the help of Zetasizer Nano ZS (Malvern, United Kingdom). The morphology of nanoparticles was visualized through transmission electron microscopy (TEM) (JEOL-2010 microscopy, Tokyo, Japan). The colloidal stability was investigated by incubating in phosphate buffered saline (PBS) with $10 \%$ fetal bovine serum (FBS) and analyzing the size of nanoparticles.

\section{Gel Retardation Assay}

The integrity of nanoparticles after 7 days' incubation in PBS with $10 \%$ BSA was investigated by gel retardation assay. Naked siRNA was used as a control. Samples were mixed with gel loading buffer containing $1 \%$ SDS and separated by $2 \%$ agarose 
gel electrophoresis. The samples in gel were observed by ethidium bromide staining.

\section{Immunohistochemistry}

Immunohistochemistry was employed to evaluate CSA expression in placenta tissues. After being deparaffinized and rehydrated, slides were treated by antigen retrieval buffer. Then the tissue sections were incubated with BSA for $1 \mathrm{~h}$ at room temperature. Then the sections were incubated with mouse monoclonal anti-chondroitin sulfate antibody (Abcam, Cambridge, United Kingdom) or control IgG (Abcam) at $4^{\circ} \mathrm{C}$ overnight. After being washed, the sections were incubated with HRP-donkey anti-mouse IgG (ThermoFisher, Waltham, United States). The sections were observed under a light microscope.

\section{Immunofluorescence}

Cells were fixed by $4 \%$ paraformaldehyde for $30 \mathrm{~min}$ at room temperature and permeabilized by $0.05 \%$ Triton X-100 for $10 \mathrm{~min}$. After being blocked by $5 \%$ BSA for $1 \mathrm{~h}$ at room temperature, cells were incubated with anti-chondroitin sulfate antibody (Abcam) at $4^{\circ} \mathrm{C}$ overnight. After being washed by PBS for three times, the cells were incubated with anti-mouse IgG FITC antibody (Abcam) for $1 \mathrm{~h}$ at room temperature. Cell nucleus were stained with DAPI. Cells were observed by confocal microscope.

\section{Flow Cytometry}

$5 \times 10^{4}$ HTR-8/SVneo cells were planted in each well of a 24-well plate. After $24 \mathrm{~h}$, cells were incubated with free Cy5 labeled siRNA (Cy5-siRNA), NP Cy5-siRNA, or T-NP $_{\text {Cy5-siRNA }}$ (100 nM) for $4 \mathrm{~h}$. After incubation, cells were collected and analyzed by FACSCalibur (BD Biosciences, Bedford, United States). Mean fluorescence intensity of Cy5 was calculated by FlowJo 7.6.1 software.

\section{ELISA}

fms-like tyrosine kinase 1 protein levels in cell medium or serum were evaluated by ELISA kit (R\&D Systems, Minneapolis, United States) based on the manufacturer's instructions. Experiments were done in duplicate, and the protein levels were calculated using a standard curve. The activities of alanine aminotransferase (ALT) and aspartate aminotransferase (AST) in serum of mice were analyzed by colorimetric assay kits (Sigma, St. Louis, United States) based on the manufacturer's instructions.

\section{Real-Time PCR}

Trizol reagent (Invitrogen, Waltham, MA, United States) was used for the extraction of total RNA. First Strand cDNA Synthesis Kit (Sigma, St. Louis, MO, United States) was employed to synthesis cDNA from relative RNA. Real-time PCR was executed by SYBR Green Real-Time PCR Master Mixes (ThermoFisher, Waltham, MA, United States) following manufacturer's instruction. $\beta$-actin and GAPDH were employed as internal control. All the primers were shown here:

Human sFLT-1 F: GAAATGGTGAGTAAGGAAAGC

\author{
Human sFLT-1 R: TACTGTCCCAGATTATGCGTTT \\ Human $\beta$-actin F: CTGGTGCCTGGGGCG \\ Human $\beta$-actin R: AGCCTCGCCTTTGCCG \\ Mouse sFlt-1 F: CGACTCACTATAGGGAGACCC \\ Mouse sFlt-1 R: TGGCCTGCTTGCATGATGTGCTGG \\ Mouse GAPDH F: GTGGCAAAGTGGAGATTGTTGCC \\ Mouse GAPDH R: GATGATGACCCGTTTGGCTCC
}

\section{High-Performance Liquid Chromatography (HPLC)}

High-performance liquid chromatography was employed to evaluate the amount of sisFLT1 accumulated in different tissues. Tissues were harvested 1 day after injection. The performance of HPLC was based on the standard method (Zhang et al., 2018). One day after injection, relative tissues were collected and homogenized in PBS. $10 \%$ perchloric acid was added into the mixture and vortexed for $3 \mathrm{~min}$. After being centrifuged at $14,000 \times g$ for $20 \mathrm{~min}$, supernatants were collected and filtered $(0.45 \mu \mathrm{m})$. Mobile phase was composed of $40 \mathrm{mM}$ potassium phosphate dibasic and acetonitrile. Flow rate was $1.0 \mathrm{~mL} / \mathrm{min}$. Ultraviolet absorbance at $313 \mathrm{~nm}$ was detected.

\section{Statistical Analysis}

Statistical analysis was performed by GraphPad PRISM 6.0 software. Data were presented as mean \pm SD. Student's $t$-test, one or two-way ANOVA analysis with a post hoc test were used to calculate the differences. Statistical analysis was significant when $P$ value $<0.05$.

\section{RESULTS}

\section{The Preparation and Characterization of Trophoblast-Targeted sFLT1 siRNA-Encapsulated Nanoparticles}

As shown in Figure 1A, $\mathrm{NP}_{\text {sisFLT1 }}$ was constructed from COOH-PEG 5 K-PLA 8 K, DOTAP, and sisFLT1 through double

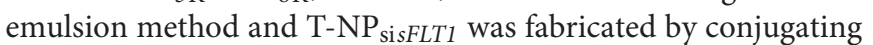
P-CSA-BP to the surface of $\mathrm{NP}_{\text {sisFLT1 }}$. DLS results shown that the resulted nanoparticles $\mathrm{T}-\mathrm{NP}_{\text {sisFLT1 }}$ had a relative larger particle size than $\mathrm{NP}_{\text {sisFLT1 }}$ (Figure 1B). The conjugation of P-CSA-BP enlarged the size of the nanoparticle. The morphology of these two nanoparticles was checked by TEM and the representative TEM images were shown in Figure 1C. The P-CSA-BP could be observed on the surface of

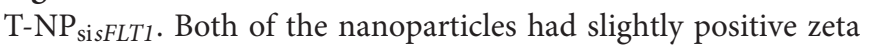
potentials (Figure 1D). The incubation of siRNA-encapsulated nanoparticles in PBS demonstrated the colloidal stability of these two nanoparticles (Figure 1E). Based on the results of agarose gel retardation assay, no significant siRNA release was observed, indicating that both nanoparticles were successfully protected the encapsulated siRNA (Figure 1F). So, nanoparticles $\mathrm{T}_{-} \mathrm{NP}_{\text {sisFLT } 1}$ was successfully generated from $\mathrm{NP}_{\text {sisFLT } 1}$ and was proved to be stable. 


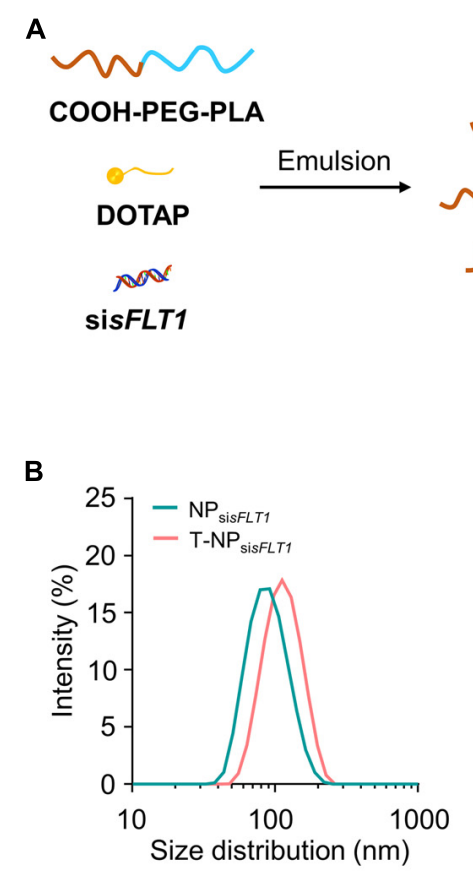

D

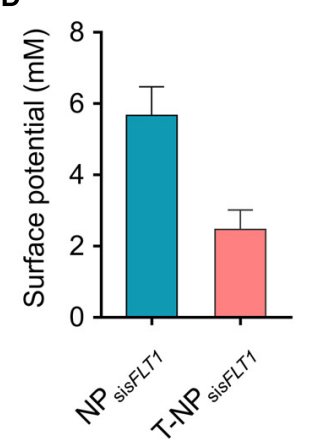

E

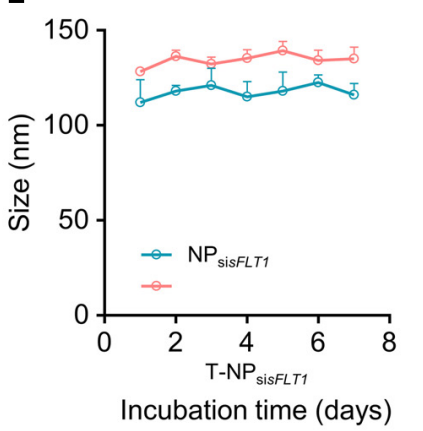

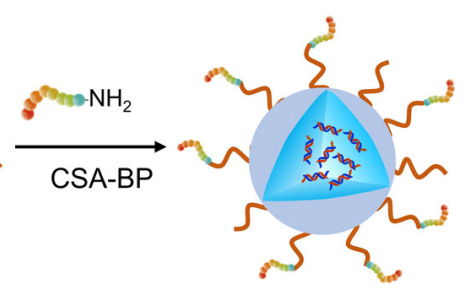

${\mathrm{T}-N P_{\text {sisFLT1 }}}$

$$
\mathrm{NP}_{\text {sisFLT1 }}
$$

C

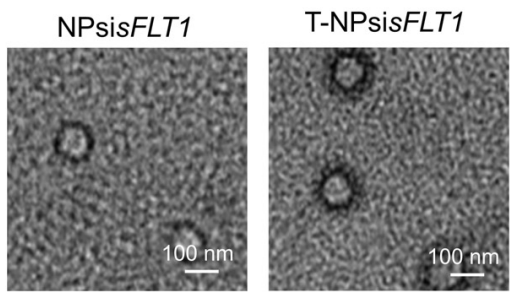

$\mathbf{F}$
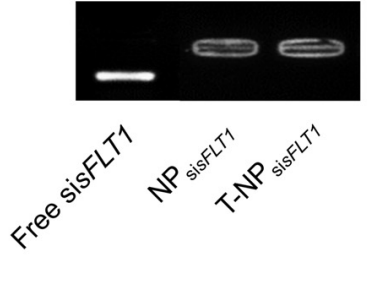

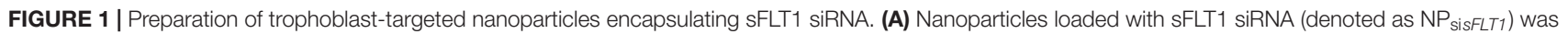
constructed from $\mathrm{COOH}-\mathrm{PEG}_{5 \mathrm{~K}}-\mathrm{PLA} \mathrm{A}_{8 \mathrm{~K}}$, cationic lipid DOTAP, and sisFLT1 using double emulsion method. Trophoblast-targeted nanoparticles (denoted as

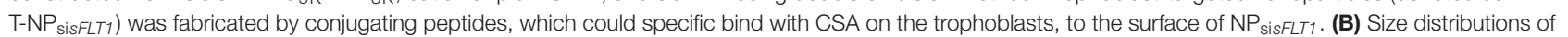

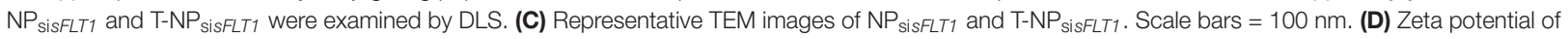
nanoparticles examined via DLS. (E) Effect of incubation in PBS on the particle size. (F) Gel retardation assay for examining the integrity of nanoparticles 7-day post construction.

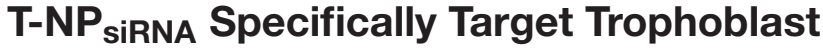 Cells in vitro}

Chondroitin sulfate $\mathrm{A}$ is a protein which is proved to exist on the surface of placental syncytiotrophoblasts. Through the immunohistochemistry, we proved the expression of CSA in the tissue of mouse placenta (Figure 2A). HTR-8/SVneo cells were human trophoblast cells and the expression of CSA was observed through immunofluorescence (Figure 2B). To investigate the uptake of these two kinds of nanoparticles into HTR-8/SVneo cells, siRNA was labeled with Cy5 and cells were analyzed by flow cytometry after incubating with nanoparticles. Based on the result of flow cytometry, trophoblast cells incubated with T-NP ${ }_{\text {Cy5-siRNA }}$ had a significantly higher Cy5 signal intensity than those incubated with $\mathrm{NP}_{\mathrm{Cy} 5 \text {-siRNA }}$ (Figures 2C,D). Furthermore, the internalized $\mathrm{NP}_{\mathrm{Cy} \text {-siRNA }}$ and T-NP $\mathrm{Cy5}_{\text {-siRNA }}$ in HTR-8/SVneo cells were observed by confocal microscopy (Figure 2E). These results indicated that $\mathrm{T}-\mathrm{NP}_{\text {siRNA }}$ had a higher efficiency than $\mathrm{NP}_{\text {siRNA }}$ in targeting to trophoblast cells in vitro.

\section{T-NP sisfLT1 Inhibit the Expression of sFLT1 in vitro}

To demonstrate the function of these two sFLT1 siRNAencapsulated nanoparticles in the inhibition of sFLT1 expression in vitro, HTR-8/SVneo cells were treated with free $\mathrm{si}_{\text {SFLT1 }}$, $\mathrm{NP}_{\text {SisFLT1 }}$ or T-NP $\mathrm{Ni}_{\text {sisLT1 }}$ for $48 \mathrm{~h}$. The mRNA levels of sFLT1 in cells were evaluated through RT-PCR and the results were shown in Figure 3A. When compared with cells treated by $\mathrm{NP}_{\text {SisFLT1 }}$, those cells treated by $\mathrm{T}-\mathrm{NP}_{\text {SisFLT1 }}$ shown a significant 
A

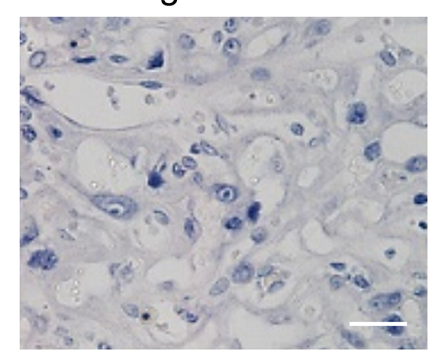

C

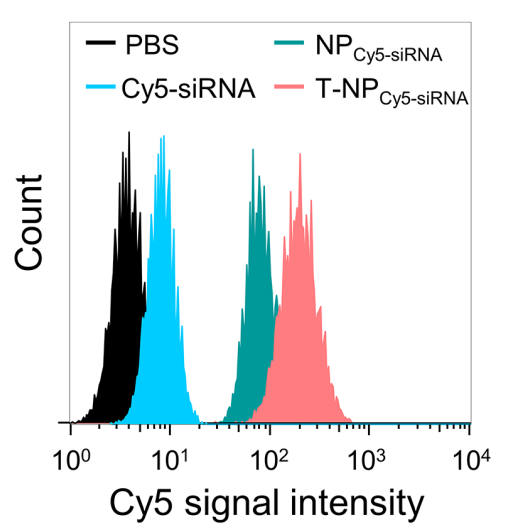

Anti-CSA

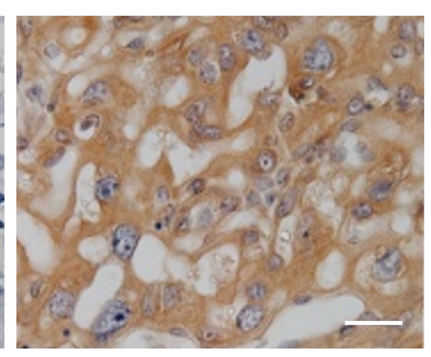

D

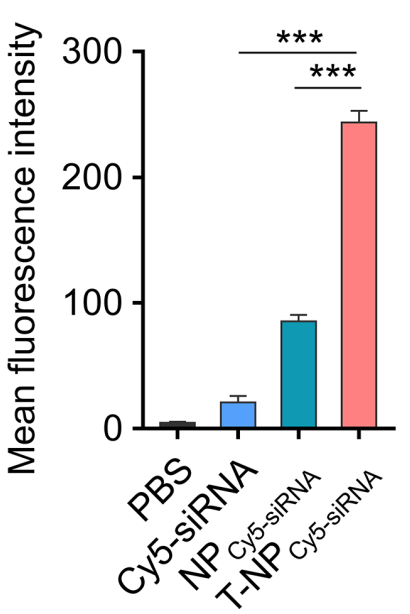

B

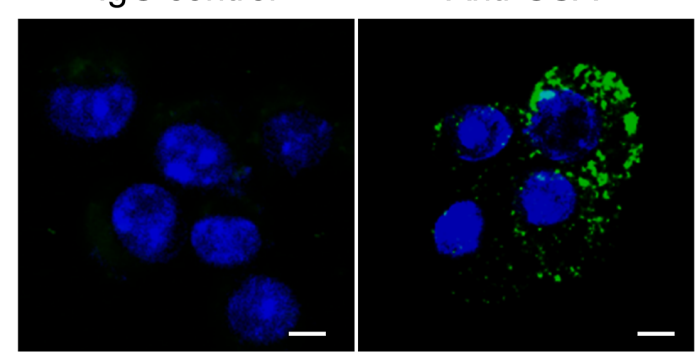

Blue: Cell nuclei Green: CSA

E

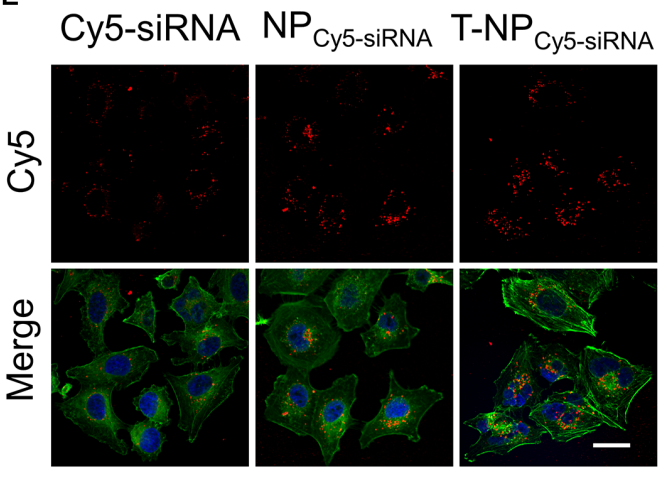

Green: F-actin Blue: Cell nuclei Red: Cy5

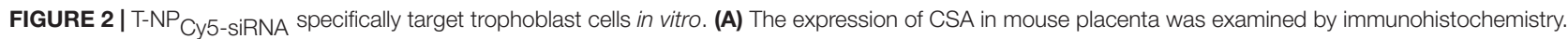
Scale bar $=20 \mu \mathrm{m}$. (B) The expression of CSA on HTR-8/SVneo cells was examined by confocal microscopy. CSA was stained with IgG isotype control antibody or anti-CSA primary antibody and anti-mouse IgG (whole molecule) FITC antibody. Scale bar $=10 \mu \mathrm{m}$. (C) Flow cytometry analyses of HTR-8/SVneo cells after $4 \mathrm{~h}$

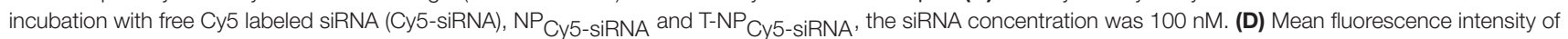

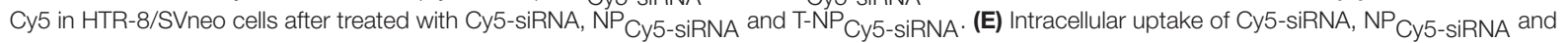
T-NP Cy5-siRNA were examined by confocal microscopy. Cell nuclei were stained with DAPI; the cytoskeleton was stained with Alexa Fluor 488 Phalloidin. Scale bar $=20 \mu \mathrm{m}$. Data was shown as mean $\pm \mathrm{SD}$. ${ }^{* \star} p<0.001$.

lower sFLT1 mRNA level and this phenomenon was enhanced by the increasing dosage. The protein level of sFLT1 was evaluated by ELISA and shown the same tendency with mRNA (Figure 3B). The protein levels of sFLT1 in HTR-8/SVneo cells were not altered by the treatment of nanoparticles encapsulating nonsense siRNA $\left(\mathrm{NP}_{\text {siNC }}\right.$ and $\left.\mathrm{T}-\mathrm{NP}_{\text {siNC }}\right)$ (Figure $3 \mathrm{C}$ ). $\mathrm{T}^{-\mathrm{NP}_{\text {sisFLT1 }} \text { had }}$ a higher efficiency than $\mathrm{NP}_{\text {sisFLT1 }}$ in the inhibition of sFLT1 expression in trophoblast cells in vitro.

\section{T-NP ${ }_{\text {sisfLT1 }}$ Selectively Accumulate in Placenta and Downregulate sFLT1 Expression in Mice}

The function of T-NP $\mathrm{TisLT1}_{\text {s }}$ was further checked in mouse model. Experimental design for testing accumulation ability of

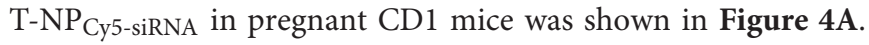
To illustrate the accumulation of the nanoparticles, pregnant $\mathrm{CD} 1$ mice were intravenously injected with $\mathrm{NP}_{\text {Cy5-siRNA }}$ and

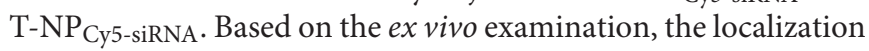

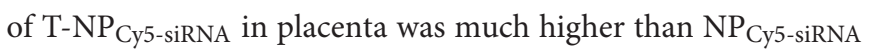
(Figure 4B). HPLC was employed to quantitatively compare the accumulation of the nanoparticles in different organs. As shown in Figure 4C, the amount of Cy5-siRNA in the placenta of mice treated by $\mathrm{T}^{-N} \mathrm{Cy5}_{\text {-siRNA }}$ was significantly higher than in the placenta of mice treated by $\mathrm{NP}_{\text {Cy5-siRNA. But the distribution }}$ of Cy5-siRNA shown no significant differences in other organs.

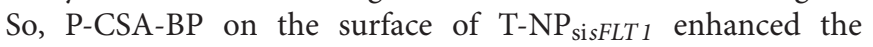
nanoparticle's specific accumulation ability in mice placenta. Experimental design for testing silencing ability of T-NP sisFLT1 $_{1}$ in pregnant CD1 mice was shown in Figure 4D. We examined the mRNA levels of sFLT1 in the placenta of mice treated by different nanoparticles through RT-PCR. When compared with $\mathrm{NP}_{\text {sisFLT1 }}$, the treatment of T-NP ${ }_{\text {sisFLT1 }}$ significantly decreased the mRNA level of sFLT1 in the placenta of mice (Figure 4E). Circulating sFLT1 levels in mice were evaluated by ELISA and shown the same tendency (Figure $4 \mathbf{F}$ ). So, P-CSA-BP on the surface of T-NPsisFLT1 also enhanced the nanoparticle's sFLT1 silencing ability in mice placenta. It was proved that $\mathrm{T}-\mathrm{NP}_{\text {sisFLT1 }}$ 

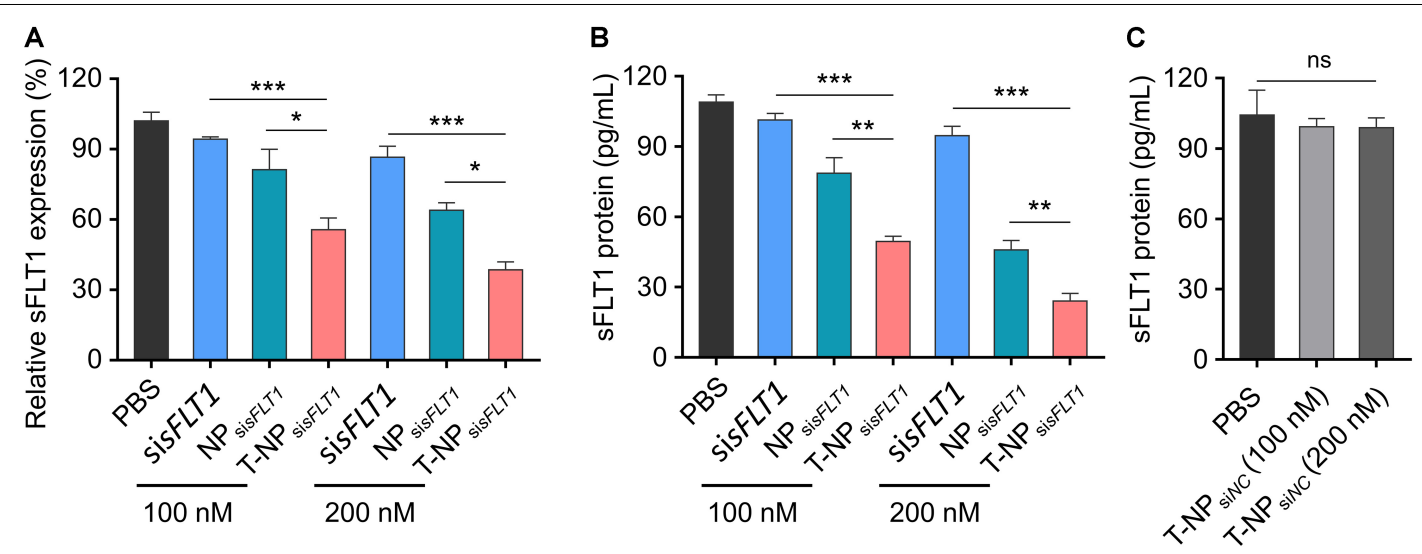

FIGURE 3 | Efficient silencing of sFLT1 by T-NP sisFLT1 in vitro. (A) Silencing of sFLT1 by different formulations in a dose-dependent manner. HTR-8/SVneo cells were

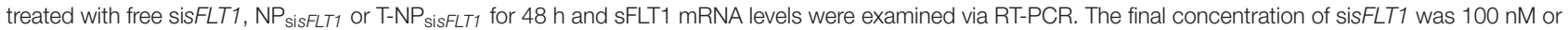
$200 \mathrm{nM}$ in present study. (B) sFLT1 protein levels produced by HTR-8/SVneo cells treated with different formulations for $48 \mathrm{~h}$. sFLT1 protein levels were measured by

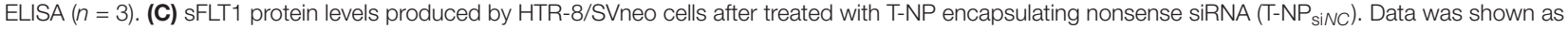
mean \pm SD. ${ }^{\star} p<0.05,{ }^{\star \star} p<0.01,{ }^{\star \star \star} p<0.001$. ns. no significant difference.

had a higher efficiency than $\mathrm{NP}_{\text {sisFLT1 }}$ in accumulation in the placenta of mice and silencing sFLT1.

\section{Conjugating P-CSA-BP to the Surface of NP $_{\text {sisflT1 }}$ Does Not Enhance the Toxic Effect}

We further investigated whether conjugating P-CSA-BP to the surface of $\mathrm{NP}_{\text {sisFLT1 }}$ enhanced the nanoparticle's toxic effect in mice. The changes of ALT and AST were widely used for illustrating the severity of the toxic effect on liver. The levels of the maternal liver transaminases ALT and AST in mice was analyzed after treatment. The normal ALT and AST levels indicated that

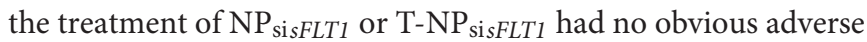
effects (Figures 5A,B). We also checked the influence of these nanoparticles on the fetus. Both newborn pup number and weight were not influenced by the treatment of $\mathrm{NP}_{\text {sisFLT1 }}$ or

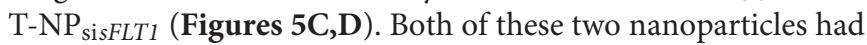
low maternal and fetal toxic effect. Thus, conjugating P-CSA-BP to the surface of NPsisFLT1 did not enhance the nanoparticle's toxic effect in mice.

\section{DISCUSSION}

Based on previous research, the pathogenesis of $\mathrm{PE}$ can be divided into two different stages (Redman and Sargent, 2009). The first stage is asymptomatic, but abnormal placenta formation and accumulation of placenta released factors in maternal circulation can be observed. The symptomatic second stage is characterized by placental oxidative stress, hypertension and proteinuria, that eventually result in angiospasm in brain which caused eclampsia (Hladunewich et al., 2007). Since the molecular mechanism during the pathogenesis of PE is not fully understand, PE still lacks reliable method for prediction and diagnosis and effective therapeutic strategy (Ahmed et al., 2017). Untreated PE is life threatening and will develops into eclampsia with several severe complications (Arulkumaran and Lightstone, 2013). Till now, the only effective therapy for PE is the early delivery of the pregnancy.

In recent years, the pathogenesis of $\mathrm{PE}$ is proved to have a strong correlation with the factors generated and released from placenta (Levine et al., 2004). Several different researches have demonstrated the abnormal increased level of anti-angiogenic molecule sFLT-1 in the circulation of women with PE (He et al., 1999; Maynard et al., 2003). The accumulation of sFLT1 has a strong correlation with the generation of hypertension through its function in antagonizing VEGF and PlGF (Fan et al., 2014). The sFLT-1/PlGF ratio is reported to be useful for the diagnosis and prognosis assessment of PE (Herraiz et al., 2018). sFLT-1 is also considered as a potential PE therapeutic target. Thadhani et al. (2016), have proved that apheresis therapy is efficient in removing sFLT-1 in the circulation of women with $\mathrm{PE}$ and extends the pregnancies with no obvious adverse effects on both mother and fetus. Brownfoot et al. (2016), demonstrated that metformin inhibits the production of sFLT-1 in villous trophoblast and prevents the pathogenesis of PE. In both mouse and baboon PE model, systemically delivered modified sFLT1 siRNAs accumulate in placentas, inhibit sFLT1 expression, and alleviate hypertension and proteinuria (Turanov et al., 2018). RNAi-based placental sFLT1 expression modulation is proved to be a novel therapeutic approach for PE. However, several limitations still exist in the development of this therapeutic strategy, such as siRNA degradation and difficulty in crossing cell membrane (Liu et al., 2014).

Nanoparticles can act as delivery system and have several benefits, including enhanced permeability, retention effect, and extravasation ability and are widely used in the therapy of cancer and other disease (Cheng et al., 2015). The transfer ability of nanoparticles to different organs is critical for the therapy. It is reported that molecular shape, surface modification, and particle size determine whether the nanoparticle can cross the placenta barrier (Muoth et al., 2016). Poly-amidoamine (PAMAM) dendrimers are shown to be excellent nanocarrier for 


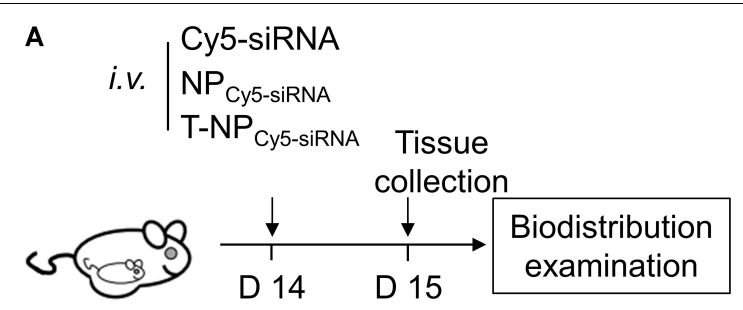

B

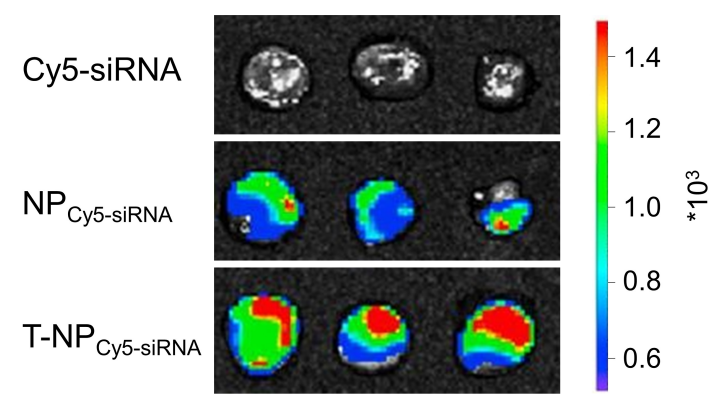

C

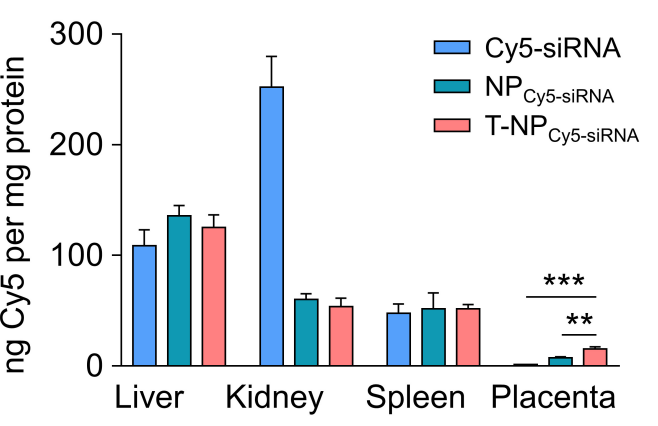

D

E

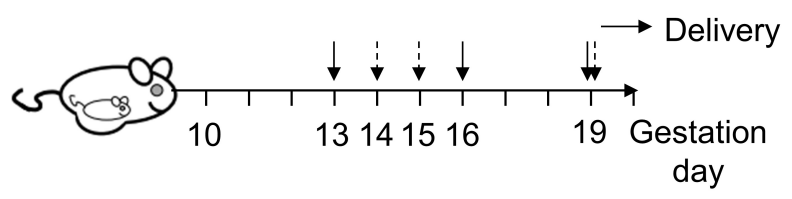

$\downarrow$ Treatment $\downarrow$ Blood collection $\downarrow \downarrow$ Tissue collection

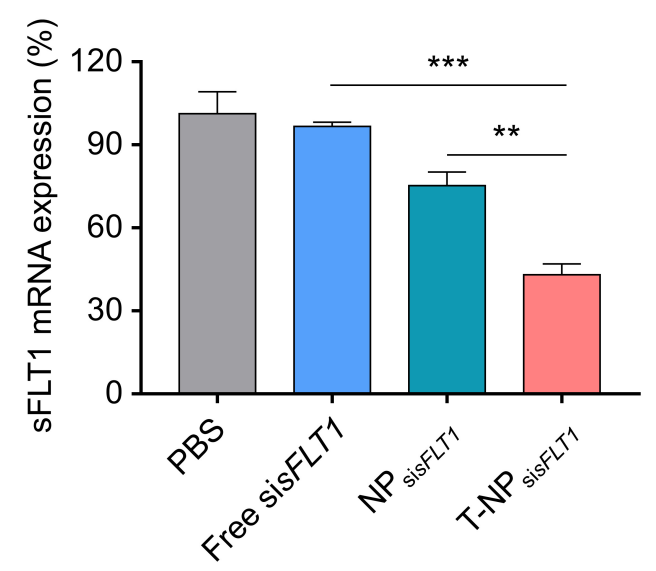

$\mathbf{F}$

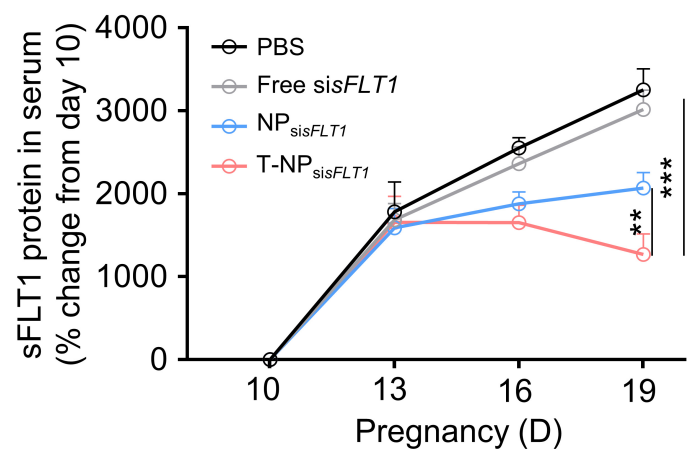

FIGURE 4 | T-NP sisFLT1 $_{1}$ selectively accumulate in placenta and downregulate SFLT1 expression. (A) Experimental design for in vivo testing of T-NP siRNA $_{\text {accumulation }}$ in pregnant CD1 mice. Pregnant CD1 mice treated with T-NP encapsulating Cy5-labeled sisFLT1 at 14 days of gestation, and the injection dosage of Cy5-sisFLT1

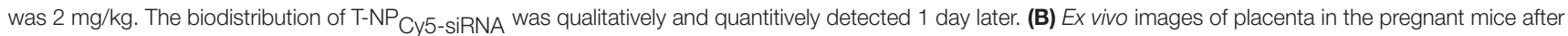
Cy5-labled siRNA injection at 15 days of gestation. (C) Amount of Cy5 accumulated in indicated tissues 1 day after injection using HPLC. (D) Experimental design for in vivo silencing of SFLT1 via NP sisFLT1. Pregnant CD1 mice were intravenously injected with $2 \mathrm{mg} / \mathrm{kg}$ of sisFLT1 on pregnancy days 14 and 15 days of gestation, and killed for examination on allowed to deliver pups on 19 days of gestation or allowed to delivery. Blood was collected on the indicated days. (E) sFLT1 mRNA expression in placenta on 19 days of gestation examined via RT-PCR ( $n=8$ biologically independent animals). (F) Circulating sFLT1 levels from mice injected with free

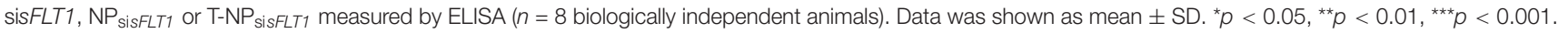

siRNA delivery and siRNA-sFLT1-PAMAM complex is proved to be effective in inhibition sFLT1 expression and improving pregnancy outcomes in PE rat model (Yu et al., 2017). PEG-PLA nanoparticle generated by double-emulsion methods is a novel siRNA delivery system which is proved in both in vivo and in vitro cancer models (Yang et al., 2011). We aimed to use this PEGPLA nanoparticular system for the delivery of sFLT1 siRNA into placenta and inhibiting the pathogenesis of PE.
Despite the placenta is unrestricted exposed to drugs in maternal circulation, only a small proportion of non-targeted nanoparticles are successfully transported into placenta (Zhang et al., 2018). Thus, specific targeting the nanoparticles to placental tissues is critical for the development of nanoparticle-based therapeutic strategy for pregnancy-related disorders. CSA is a protein which exist on placental syncytiotrophoblasts. We confirmed the existence of CAS in both mice placenta tissues 

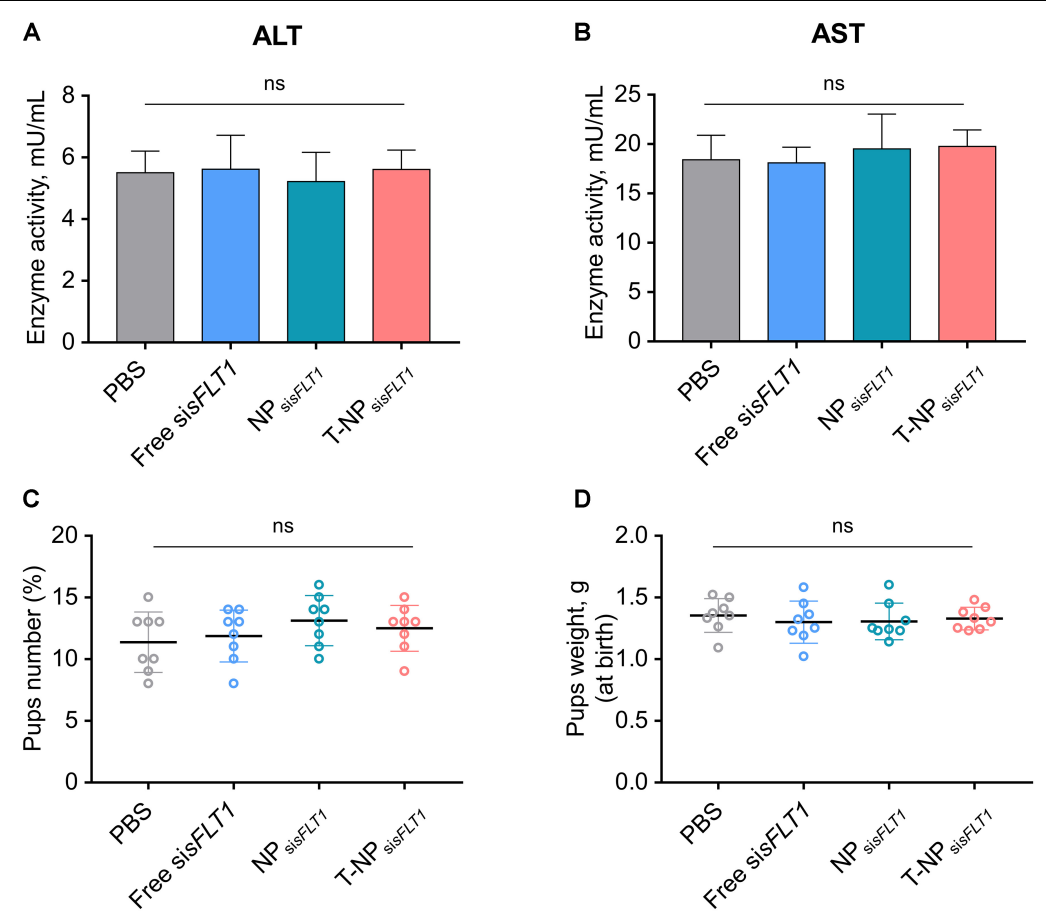

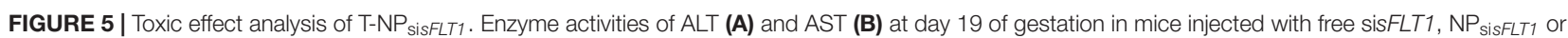

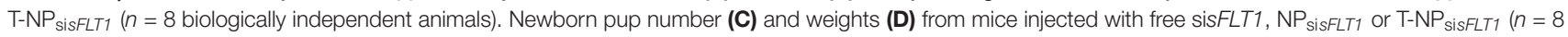
biologically independent animals). ns. no significant difference.

and human trophoblast HTR-8/SVneo cells. Synthetic placental CSA-binding peptide can specifically bind to trophoblasts in placenta but not to other cells expressing CSA in other tissues. It is reported that P-CSA-BP-conjugated nanoparticles is effective in targeting to the placenta and is a novel placentaspecific drug delivery option (Zhang et al., 2018). Based on the PEG-PLA nanoparticular system, we synthesized P-CSABP-conjugated PEG-PLA nanoparticles encapsulating sFLT1 siRNA and analyzed its function both in vivo and in vitro. Conjugating P-CSA-BP enlarged the particle size and decreased the surface potential of PEG-PLA nanoparticles. Both $\mathrm{NP}_{\text {sisFLT1 }}$ and T-NP $\mathrm{NiSFLT1}_{1}$ nanoparticles were proved to be stable in PBS and shown strong ability in the protection of encapsulated siRNA. HTR-8/SVneo cells were generated from isolated first trimester extravillous cytotrophoblasts through the infection with simian virus 40 large $\mathrm{T}$ antigen (Abou-Kheir et al., 2017). In human trophoblast HTR-8/SVneo cells, the existence of P-CSA$\mathrm{BP}$ on the surface of nanoparticles significantly enhanced its accumulation ability, which was shown by both flow cytometry analyses and immunofluorescence results. We also checked the efficiency of $\mathrm{NP}_{\text {SisFLT1 }}$ and $\mathrm{T}-\mathrm{NP}_{\text {SisFLT1 }}$ nanoparticles in targeting to the placentas of pregnant CD1 mice. Ex vivo images and HPLC results demonstrated that nanoparticles with P-CSA-BP on the surface had a significantly stronger ability in accumulation in the placenta of mice. So, conjugating P-CSA-BP to the surface of PEG-PLA nanoparticles enhanced its ability in targeted delivery of drugs to the placenta.

In this research, we aimed to decrease the expression of sFLT1 in placenta through the trophoblast-targeted nanoparticles encapsulating sFLT1 siRNA. In HTR-8/SVneo cells, T-NP ${ }_{\text {sisFLT1 }}$ nanoparticles had a higher efficiency in inhibition of sFLT1 expression than $\mathrm{NP}_{\text {sisFLT1 }}$ nanoparticles, which was shown in both mRNA and protein levels. Also, after being injected into pregnant CD1 mice, $\mathrm{T}-\mathrm{NP}_{\text {sisFLT1 }}$ nanoparticles significantly decreased the sFLT1 mRNA level in placenta and sFLT1 protein level in serum. Trophoblast-targeted sFLT1 siRNA-encapsulated

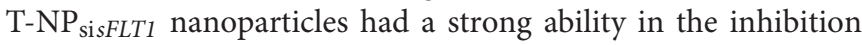
of sFLT1 expression in placenta and decreasing the amount of sFLT1 in circulation.

Nanoparticles used as delivery system in the therapy of PE must be safe for both mother and fetus. It should have no adverse effects on tissues, do not cross the placenta into fetus, and show no apparent fetal toxicity. The injection of T-NP sisFLT1 $_{1}$ nanoparticles did not influenced the enzyme activities of ALT and AST in pregnant mice. Meanwhile, the newborn pups number and weights were also not changed. These results illustrated that

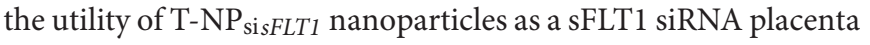
specific delivery system was safe in mice.

In order for this placenta targeted nanoparticles to be adopted and accepted clinically, further research and development are required. The maximum dosage of this nanoparticles should be tested. The nanoparticles should be optimized to improve the efficiency in encapsulating and releasing sFLT1 siRNA. More toxicity tests should be performed in different animal models to ensure the safety of this nanoparticles. Although we had demonstrated sFLT1 silencing function of T-NPsisFLT1 nanoparticles in mouse placenta, but its effects in the prevention or therapy against $\mathrm{PE}$ in mice was not investigated. So, 
further studies should be done to demonstrate the function of T-NPsisFLT1 nanoparticles in PE therapy.

In conclusion, the employment of T-NP ${ }_{\text {sisFLT1 nanoparticles }}$ as a sFLT1 siRNA placenta specific delivery system significantly silenced sFLT1 in mice and was safe for both the mother and fetus. This nanoparticle might serve as a novel potential therapeutic strategy for PE.

\section{DATA AVAILABILITY STATEMENT}

The raw data supporting the conclusions of this article will be made available by the authors, without undue reservation, to any qualified researcher.

\section{ETHICS STATEMENT}

The animal study was reviewed and approved by the Shandong Provincial Hospital Affiliated to Shandong University.

\section{REFERENCES}

Abou-Kheir, W., Barrak, J., Hadadeh, O., and Daoud, G. (2017). HTR-8/SVneo cell line contains a mixed population of cells. Placenta 50, 1-7. doi: 10.1016/ j.placenta.2016.12.007

Ahmad, S., and Ahmed, A. (2004). Elevated placental soluble vascular endothelial growth factor receptor-1 inhibits angiogenesis in preeclampsia. Circ. Res. 95, 884-891. doi: 10.1161/01.RES.0000147365.86159.f5

Ahmed, A., Rezai, H., and Broadway-Stringer, S. (2017). Evidence-based revised view of the pathophysiology of preeclampsia. Adv. Exp. Med. Biol. 956, 355-374. doi: 10.1007/5584_2016_168

Anderson, U. D., Olsson, M. G., Kristensen, K. H., Akerstrom, B., and Hansson, S. R. (2012). Review: biochemical markers to predict preeclampsia. Placenta 33, S42-S47. doi: 10.1016/j.placenta.2011.11.021

Arulkumaran, N., and Lightstone, L. (2013). Severe pre-eclampsia and hypertensive crises Best. Pract. Res. Clin. Obstet. Gynaecol. 27, 877-884. doi: 10.1016/j.bpobgyn.2013.07.003

Brownfoot, F. C., Hastie, R., Hannan, N. J., Cannon, P., Tuohey, L., Parry, L. J., et al. (2016). Metformin as a prevention and treatment for preeclampsia: effects on soluble fms-like tyrosine kinase 1 and soluble endoglin secretion and endothelial dysfunction. Am. J. Obstet. Gynecol. 214, e351-e356. doi: 10.1016/j. ajog.2015.12.019

Cheng, C. J., Tietjen, G. T., Saucier-Sawyer, J. K., and Saltzman, W. M. (2015). A holistic approach to targeting disease with polymeric nanoparticles. Nat. Rev. Drug Discov. 14, 239-247. doi: 10.1038/nrd4503

Douglas, K. A., and Redman, C. W. (1994). Eclampsia in the United Kingdom. BMJ 309, 1395-1400. doi: 10.1136/bmj.309.6966.1395

Fan, X., Kambham, N., Sung, J. F., Singh, N., Petitt, M., Dhal, S., et al. (2014). Endometrial VEGF induces placental sFLT1 and leads to pregnancy complications. J. Clin. Invest. 124, 4941-4952. doi: 10.1172/JCI76864

He, H., Venema, V. J., Gu, X., Venema, R. C., Marrero, M. B., and Caldwell, R. B. (1999). Vascular endothelial growth factor signals endothelial cell production of nitric oxide and prostacyclin through flk-1/KDR activation of c-Src. J. Biol. Chem. 274, 25130-25135. doi: 10.1074/jbc.274.35.25130

Henderson, J. T., Whitlock, E. P., O'Connor, E., Senger, C. A., Thompson, J. H., and Rowland, M. G. (2014). Low-dose aspirin for prevention of morbidity and mortality from preeclampsia: a systematic evidence review for the U.S. Prevent. Serv. Task Force Ann. Intern. Med. 160, 695-703. doi: 10.7326/M132844

Herraiz, I., Llurba, E., Verlohren, S., Galindo, A., and Spanish Group for the Study of Angiogenic Markers in Preeclampsia. (2018). Update on the diagnosis and prognosis of preeclampsia with the Aid of the sFlt-1/ PlGF ratio in

\section{AUTHOR CONTRIBUTIONS}

LL, HY, and PC conceived the study. TX, QZ, DW, and YZ designed the experiments and analyzed the data. SW secured the funding and supervised the project.

\section{FUNDING}

This study was supported by the Key Research \& Development Project of Shandong Province (2019GSF108096), the National Natural Science Foundation of China (81801473, 81971409, 81741037, 81741038, and 81100447), the Shandong Medical and Health Technology Development Program of China (2016WS0412), the Chongqing Postdoctoral Research Special Funding Project (XmT2018077), the China Postdoctoral Science Foundation (2018M631067 and 2019T120814), the Open Project of NHC Key Laboratory of Fertility Control Technology (2018KF002), and the Jinan Science and Technology Program (201907011 and 201907012).

singleton pregnancies. Fetal. Diagn. Ther. 43, 81-89. doi: 10.1159/00047 7903

Hladunewich, M., Karumanchi, S. A., and Lafayette, R. (2007). Pathophysiology of the clinical manifestations of preeclampsia. Clin. J. Am. Soc. Nephrol. 2, 543-549. doi: 10.2215/CJN.03761106

Laresgoiti-Servitje, E. (2013). A leading role for the immune system in the pathophysiology of preeclampsia. J. Leukoc. Biol. 94, 247-257. doi: 10.1189/jlb. 1112603

Levine, R. J., Campos-Galicia, I., Berumen-Lechuga, M. G., Molina-Pérez, C. J., García-Paleta, Y., Isordia-Salas, I., et al. (2004). Circulating angiogenic factors and the risk of preeclampsia. N. Engl. J. Med. 350, 672-683. doi: 10.1056/ NEJMoa031884

Levine, R. J., Lam, C., Qian, C., Yu, K. F., Maynard, S. E., Sachs, B. P., et al. (2006). Soluble endoglin and other circulating antiangiogenic factors in preeclampsia. N. Engl. J. Med. 355, 992-1005. doi: 10.1056/NEJMoa055352

Liu, C., Liu, X., Rocchi, P., Qu, F., Iovanna, J. L., and Peng, L. (2014). Arginineterminated generation 4 PAMAM dendrimer as an effective nanovector for functional siRNA delivery in vitro and in vivo. Bioconjug. Chem. 25, 521-532. doi: 10.1021/bc4005156

Maynard, S. E., Min, J. Y., Merchan, J., Lim, K. H., Li, J., Mondal, S., et al. (2003). Excess placental soluble fms-like tyrosine kinase 1 (sFlt1) may contribute to endothelial dysfunction, hypertension, and proteinuria in preeclampsia. J. Clin. Invest. 111, 649-658. doi: 10.1172/JCI17189

Muoth, C., Aengenheister, L., Kucki, M., Wick, P., and Buerki-Thurnherr, T. (2016). Nanoparticle transport across the placental barrier: pushing the field forward! Nanomedicine 11, 941-957. doi: 10.2217/nnm-2015-0012

Palm, M., Basu, S., Larsson, A., Wernroth, L., Akerud, H., and Axelsson, O. (2011). A longitudinal study of plasma levels of soluble fms-like tyrosine kinase 1 (sFlt1), placental growth factor (PlGF), sFlt1: PlGF ratio and vascular endothelial growth factor (VEGF-A) in normal pregnancy. Acta Obstet. Gynecol. Scand. 90, 1244-1251. doi: 10.1111/j.1600-0412.2011.01186.x

Rana, S., Powe, C. E., Salahuddin, S., Verlohren, S., Perschel, F. H., Levine, R. J., et al. (2012). Angiogenic factors and the risk of adverse outcomes in women with suspected preeclampsia. Circulation 125, 911-919. doi: 10.1161/ CIRCULATIONAHA.111.054361

Redman, C. W., and Sargent, I. L. (2009). Placental stress and pre-eclampsia: a revised view. Placenta 30(Suppl. A), S38-S42. doi: 10.1016/j.placenta.2008. 11.021

Salahuddin, S., Lee, Y., Vadnais, M., Sachs, B. P., Karumanchi, S. A., and Lim, K. H. (2007). Diagnostic utility of soluble fms-like tyrosine kinase 1 and soluble endoglin in hypertensive diseases of pregnancy. Am. J. Obstet. Gynecol. 197, e21-e26. doi: 10.1016/j.ajog.2007.04.010 
Thadhani, R., Hagmann, H., Schaarschmidt, W., Roth, B., Cingoez, T., Karumanchi, S. A., et al. (2016). Removal of soluble fms-like tyrosine Kinase-1 by dextran sulfate apheresis in preeclampsia. J. Am. Soc. Nephrol. 27, 903-913. doi: 10.1681/ASN.2015020157

Turanov, A. A., Lo, A., Hassler, M. R., Makris, A., Ashar-Patel, A., Alterman, J. F., et al. (2018). RNAi modulation of placental sFLT1 for the treatment of preeclampsia. Nat. Biotechnol. 36, 1164-1173. doi: 10.1038/nbt.4297

von Dadelszen, P., and Magee, L. A. (2014). Pre-eclampsia: an update. Curr. Hypertens. Rep. 16:454. doi: 10.1007/s11906-014-0454-8

World Health Organization [WHO] (2011). WHO Recommendations for Prevention and Treatment of Pre-Eclampsia and Eclampsia. Geneva: WHO Guidelines Approved by the Guidelines Review Committee.

Xu, C. F., Zhang, H. B., Sun, C. Y., Liu, Y., Shen, S., Yang, X. Z., et al. (2016). Tumor acidity-sensitive linkage-bridged block copolymer for therapeutic siRNA delivery. Biomaterials 88, 48-59. doi: 10.1016/j.biomaterials.2016.02.031

Yang, X. Z., Dou, S., Sun, T. M., Mao, C. Q., Wang, H. X., and Wang, J. (2011). Systemic delivery of siRNA with cationic lipid assisted PEG-PLA nanoparticles for cancer therapy. J. Control Release 156, 203-211. doi: 10.1016/j.jconrel.2011. 07.035
Yu, J., Jia, J., Guo, X., Chen, R., and Feng, L. (2017). Modulating circulating sFlt1 in an animal model of preeclampsia using PAMAM nanoparticles for siRNA delivery. Placenta 58, 1-8. doi: 10.1016/j.placenta.2017. 07.360

Zhang, B., Tan, L., Yu, Y., Wang, B., Chen, Z., Han, J., et al. (2018). Placenta-specific drug delivery by trophoblast-targeted nanoparticles in mice. Theranostics 8 , 2765-2781. doi: 10.7150/thno.22904

Conflict of Interest: The authors declare that the research was conducted in the absence of any commercial or financial relationships that could be construed as a potential conflict of interest.

Copyright (c) 2020 Li, Yang, Chen, Xin, Zhou, Wei, Zhang and Wang. This is an open-access article distributed under the terms of the Creative Commons Attribution License (CC BY). The use, distribution or reproduction in other forums is permitted, provided the original author(s) and the copyright owner(s) are credited and that the original publication in this journal is cited, in accordance with accepted academic practice. No use, distribution or reproduction is permitted which does not comply with these terms. 